\title{
La juventud de Sarajevo: redes personales e identificaciones
}

Claudia Aguilar Carretero. Departament d'Antropologia Social i Cultural (UAB) ${ }^{1}$

\section{Resumen}

En el presente artículo se presenta una investigación que analiza redes personales, identificación y juventud de Sarajevo. En primer lugar vamos a hacer una aproximación a la juventud actual de Sarajevo, en segundo lugar hablaremos de la identidad e identificación en general y específicamente entre la juventud bosnia y por ultimo vamos a introducir nuestra propuesta de identificación relacional como posible salida al bloqueado tema identitario en Bosnia y Hercegovina.

Palabras clave: Redes Personales - Identidad - Sarajevo - Juventud.

\section{Abstract}

In this article we investigate personal networks, identification and youth of Sarajevo. First we make an approach to the current situation of young people in Sarajevo, second we talk about identity and identification in genaral and specifically among young people in Sarajevo and finally we introduce our proposal of relational identification as a possible way out of the blocked identitary subject in Bosnia and Hercogovina

Key words: Personal Networks - Identity - Sarajevo - Youth.

\section{I ntroducción al contexto de estudio: Sarajevo}

Bosnia y Hercegovina constituye una realidad social bastante compleja, una de cuyas características principales es una distancia notable entre el mundo urbano y el rural. Mientras que la ciudad se ha secularizado cada vez más (por lo menos hasta antes de la última guerra 1992-1995) en los pueblos y áreas rurales perdura un modo de hacer y un pensamiento mucho más tradicional (Bringa, 1993).

Sarajevo es el principal centro urbano de Bosnia y ha asumido desde siempre un significado simbólico en el imaginario colectivo. Desde su fundación en el siglo XV, durante la ocupación otomana, ha estado siempre habitada por una población eslava religiosamente mixta compuesta de musulmanes, ortodoxos y católicos a los que se añadieron una comunidad de judíos sefarditas provenientes de la España de los Reyes Católicos. La convivencia entre las diversas religiones ha estado siempre garantizada por unos sentimientos religiosos lejanos al fundamentalismo. Sobre la base de esta convivencia se ha edificado una fuerte identidad común entre los residentes de Sarajevo, que muestran un intenso sentido de pertenencia supraétnico a la ciudad testimoniado por la alta presencia de matrimonios mixtos, que antes de la guerra llegaban al 40\% (Donia y Fine, 1994). 
Esta convivencia secular fue el principal objetivo de la violencia nacionalista de la última guerra cuyas consecuencias han afectado fuertemente la antigua manera de ser de la ciudad. Sarajevo fue condenada por lo que simbolizaba históricamente y por lo que todavía constituía; un testimonio real de la posibilidad de convivencia supra-étnica.

Los movimientos de población durante y después de la guerra han afectado sensiblemente la composición social de Sarajevo, hasta el punto de que sus propios habitantes afirman no reconocer su propia ciudad.

Se suele contraponer un anterior cosmopolitismo y florecimiento cultural con el proceso reciente de tradicionalización o "primitivización", tal como lo denomina informalmente la gente de la ciudad. Esto se debe a la asimetría en los procesos de emigración e inmigración de la ciudad durante la guerra: pudieron huir aquellos con más recursos económicos y hubo una verdadera fuga de cerebros (artistas, intelectuales, etc.) y, en cambio, los nuevos pobladores son en su mayoría población rural que ha huido o ha sido expulsada de sus casas, principalmente de Bosnia oriental. Asimismo, durante y después de la guerra una gran proporción de no-musulmanes (ortodoxos, judíos, etc.) abandonaron la ciudad y muy pocos han vuelto con lo que se puede observar una creciente "musulmanización" de la ciudad. ${ }^{2}$

Pese a ello, Sarajevo sigue siendo la ciudad más cosmopolita y tolerante de una zona en la que, bajo la mirada de una observadora externa, es fácil percibir una considerable diversidad de gentes y modos de vida. Un ejemplo de ello es que, mientras que durante el día sorprende ver un número cada vez más elevado de mujeres con velo y hombres con larga barba, por la noche los bares y pubs se llenan de jóvenes vestidos según la moda centro-europea compartiendo cervezas al son de la canción del verano.

\subsection{Juventud actual en Sarajevo}

El panorama que se les presenta a los jóvenes no es muy alentador. Según un informe de las Naciones Unidas sobre juventud ${ }^{3}$ el $65 \%$ de los jóvenes afirma que se iría del país si pudiese. En los últimos 10 años aproximadamente 100.000

\footnotetext{
${ }^{1}$ Enviar correspondencia a: Claudia Aguilar (claudia.aguilar@uab.es)

${ }^{2}$ Antes de la guerra y según el censo de 1991 los musulmanes representaban el 49\% de la población de Sarajevo. Ahora no se sabe con exactitud y hay un baile de cifras en torno a este tema pero oscila alrededor del $90 \%$
} 
jóvenes se han ido del país ${ }^{4}$. Una de las principales razones para irse del país son las escasas posibilidades de trabajo. Las posibilidades de encontrar un buen trabajo son mínimas con lo que muchos jóvenes se mueven en el sector informal de la economía. Los salarios además no resultan demasiado motivadores, con los que la salida "fácil" es buscar suerte en otros lugares.

La excepción son los que encuentran trabajo en una de las múltiples organizaciones internacionales que hay en Sarajevo. Trabajar en una de ellas comporta por un lado un trabajo no muy seguro, ya que los contratos son precarios y temporales, pero por otro lado los salarios son más altos que la media. Esta situación ha supuesto la creación de una clase media engañosa y temporal.

La participación política o el simple interés por la misma es muy minoritaria. La actitud general es quejumbrosa y descontenta para con el país y sus gobernantes corruptos, unida a una pasividad probablemente derivada de años de régimen comunista. Pocos jóvenes están implicados activamente en organizaciones o partidos políticos. Los partidos que se hallan el poder son los nacionalistas y no existe por el momento ninguna alternativa que motive, en la mayoría de jóvenes, la simple acción de ir a votar.

La vivencia de la religión en Sarajevo es similar a la de cualquier ciudad europea actual. La mayoría de los jóvenes no son creyentes y no practican la religión aunque siguen unas ciertas tradiciones que provienen de la religión como por ejemplo no comer carne de cerdo los de tradición musulmana. Después de la guerra, sin embargo, sorprende una clara tendencia a la radicalización de la práctica religiosa, sobre todo entre un sector de los musulmanes. Mientras que la práctica del velo y la barba larga nunca había formado parte del I slam Bosnio ahora su uso es cada vez más frecuente. La razón de este cambio es sin duda una gran entrada de fondos para construir mezquitas y para reforzar el Islam por parte de países como Arabia Saudita que importan un Islam diverso al que se solía practicar en Bosnia.

Un problema añadido respecto a los jóvenes es que están bastante influenciados por los adultos, no se percibe salto generacional alguno entre los gustos de unos y otros (escuchan la misma música, consumen los mismos productos audiovisuales,

\footnotetext{
${ }^{3}$ UNDP (2000)

${ }^{4}$ OIA (agencia de información para la juventud). Comunicación personal.
} 
etc.), con lo que no se desarrollan demasiadas actitudes rebeldes o contestatarias hacia los discursos de generaciones precedentes. A pesar de la actitud pasiva en general, algunos, aunque pocos, están empezando a intentar cambiar las cosas y esto les permite tener contacto con nuevos conceptos y nuevos discursos alejados del nacionalismo paterno.

A pesar de estas circunstancias entre los jóvenes de Sarajevo se respira un clima alegre y jovial y su principal herramienta para afrontar el día a día de una forma más llevadera es un fino y especial sentido del humor que ha sido incluso tema central de recientes estudios (Vucetic, 2004).

\section{La construcción social de las identidades}

\subsection{I dentidad en Bosnia y Hercegovina}

Existe poca literatura sobre el conflicto bosnio desde la antropología. La mayoría de contribuciones provienen de las ciencias políticas o del estudio de las relaciones internacionales, que prestan poca atención a las sutilezas epistemológicas de conceptos como cultura, identidad o etnicidad (Campbel, 1998)

El debate entre los autores / as que tratan la identidad en Bosnia gira en torno a la ontología de tales identidades. Las posturas adoptadas van desde los que postulan que las identidades diferenciadas han existido siempre como una constante a lo largo de los últimos siglos y por lo tanto asentadas en unas raíces sólidas y definidas (Simic 2000; Despalatovic 2000 y Banac 1984 ente otros) hasta los que plantean que éstas han sido reinventadas y manipuladas recientemente y de forma deliberada desde las elites políticas e intelectuales con unos fines claros (Petrovic 2000, Prosic-Dvornic 2000, Hammel 1993, Kaldor 2001).

En la base de estas dos posturas se sitúan respectivamente una concepción estática o dinámica de la identidad. Para los primeros la identidad étnica constituye una marca genética inmutable que se transmite de generación en generación. Para los segundos, por el contrario, la identidad es cambiante y contingente al contexto socio-histórico en que se construye.

Bringa (1995), en la única etnografía existente sobre Bosnia, centra su análisis en la identidad. Afirma que no se puede hablar de tres identidades étnicas sino que la identidad de todos los bosnios conlleva esta experiencia de convivencia interreligiosa. Ivan Lovrenovic lo secunda en la siguiente cita: 
To be bosnian was to have a feeling for otherness, for the different as a part of the daily reality of one's most personal environment. It was this experience of the different that made possible to be Bosnian. In the new territorialization, grown from the poison of chauvinism, Bosnians have ceased to be bosnians and become just Bosniak Muslims, Serbs and Croats (Lovrenovic 2001: 209) ${ }^{5}$.

Bringa constata que las diferencias religiosas que se observan en Bosnia distan mucho de ser distintas identidades étnicas. Dentro de Bosnia se dan varios modelos de identidad colectiva, ligados a factores históricos políticos y religiosos, que en determinados contextos entran en conflicto mientras que en otros conviven.

La principal crítica efectuada por esta autora carga sobre el papel de políticos e intelectuales de la comunidad internacional que han utilizado conceptualizaciones occidentales de nación y etnia, distorsionando así las propias conceptualizaciones locales y legitimando una política de limpieza étnica.

La autora afirma que los términos "croata", "serbio" y "bosniak" no se utilizaban antes de la guerra en $\mathrm{BiH}^{6}$. En el pueblo donde ella realizó su trabajo de campo, la gente se refería a los "otros" utilizando categorías religiosas y, solamente antes y durante la guerra, los católicos y ortodoxos empezaron a autodenominarse serbios y croatas y a identificarse con los países vecinos.

Al mismo tiempo, desde el estado yugoslavo se utilizaban unas conceptualizaciones para clasificar a la gente según su religión, etiquetas que eran habituales en los diversos países socialistas. De todos modos, los conceptos utilizados han sido traducidos por los mismos grupos en el sentido occidental, alejado del que poseían en Yugoslavia. Se trata, siempre según la autora, de identidades "etnoreligiosas" que provienen de la organización otomana en millets. La gente en Bosnia, independientemente de la religión, comparte lengua, territorio y organización social y económica.

\footnotetext{
5 "Ser bosnio era tener un sentimiento para la otredad, para lo diverso como una parte de la realidad diaria en el propio entorno más personal. Era esta experiencia de lo diverso lo que hacia posible ser bosnio. En la nueva territorialización, surgida del veneno del chauvinismo, los bosnios han dejado de ser bosnios para ser unicamente bosníacos, serbios o croatas" (Traducción propia)

${ }^{6} \mathrm{BiH}$ son las siglas internacionales para Bosnia y Hercegovina
} 
De todas maneras, tal como afirman Bringa y otros autores (Bowman 1994, Gagnon 1994), durante el comunismo, sobre todo en ámbitos urbanos seculares, tales identidades religiosas perdieron sentido y las personas no las tenían en cuenta para su identidad y sus relaciones. Prueba de ello es la gran cantidad de matrimonios mixtos en las zonas urbanas, cerca del $40 \%$.

Sin embargo, después de cuatro años de Guerra e intervención internacional quien resultó ser el ganador de la batalla fue el discurso nacionalista generalizado que divide oficialmente a la gente de Bosnia en Serbios, Croatas y Bosníacos y los fuerza a situarse en unos de los tres grupos para poder ser ciudadanos. Desde entonces la sociedad quedó oficialmente dividida en los tres grupos y tal división es rigurosamente custodiada por la constitución, el estado, las leyes y la comunidad internacional.

\subsection{Nuestra propuesta de identificación relacional}

La presencia de estas categorías étnicas en el discurso político y cotidiano de las personas no significa que tales categorías tengan, desde un punto de vista académico, una existencia esencial.

Es importante distinguir, como nos propuso Bourdieu, entre las categorías de práctica y las categorías de análisis. A la hora de analizar una situación o contexto social concreto se debe evitar utilizar las mismas categorías de práctica que son utilizadas por los actores sociales pues se corre el riesgo de reificar y dar estatus científico a una practica de hecho. Nuestro objetivo será entonces analizar y criticar tales categorías. La situación en Bosnia y Hercegovina nos proporciona el escenario perfecto para llevar a cabo esta tarea.

Desde hace algunos años podemos constatar una verdadera crisis en torno a los términos tradicionales que se utilizan en antropología y demás ciencias sociales para describir como se organizan las personas en grupos sociales. Algunos científicos como Beck, Bauman o Delanty, entre otros, apuntan la necesidad de nuevas propuestas en este sentido ${ }^{7}$.

Nuestra propuesta empieza por utilizar el termino identificación en lugar del de identidad, como nos proponen Brubaker y Cooper (2000) ya que resulta menos

\footnotetext{
7 Debate surgido con los científicos mencionados en el marco del Seminario "Ausencia de Fronteras: la mirada Cosmopolita" organizado en Barcelona por el CIDOB el 9 de Marzo del 2007. El programa del seminario se puede consultar en www.cidob.org
} 
rígido y nos permite ilustrar mejor el hecho de que la identificación es un proceso social que depende del contexto y la situación. De igual manera nos permite diferenciar entre la auto-identificación y la identificación por otros.

Nuestra propuesta ${ }^{8}$ es que la identificación personal tanto la auto-identificación como la identificación por otros es relacional, es decir, se trata de un proceso continuo que tiene lugar en la red de relaciones personales y varía en función de los diversos contextos y situaciones.

Por ello hemos incluido en nuestra investigación la metodología de análisis de redes personales. Estudiar no únicamente las personas y sus discursos sino sus comunidades desterritorializadas y dispersas puede aportarnos interesante información acerca de la realidad cotidiana de la persona, que es el ámbito en el cual se negocian constantemente las identificaciones colectivas.

\section{Redes de identificación en Sarajevo}

La fase previa de este estudio, realizada en Sarajevo en el año 2003, se centró en un grupo reducido de jóvenes de la ciudad y nos permitió poner a prueba nuestra metodología.

Aquellos primeros resultados nos proporcionaron información sobre qué términos se utilizaban en Sarajevo para clasificar a las personas de BiH en diversos grupos, el uso de tales categorías, las líneas discursivas sobre la ontología de las mismas y la relación entre el uso de determinadas categorías, los discursos sobre la identificación y, por último, la etnicidad y las redes personales ${ }^{9}$.

\subsection{Objetivos}

La presente investigación persigue, en consecuencia, analizar la complejidad que envuelve todo el proceso de identificación. El estudio se centra en las redes de relaciones sociales de los jóvenes de Sarajevo y en sus discursos identitarios para captar sobre qué premisas se está construyendo la sociedad futura del nuevo país surgido de la guerra.

Tratamos de recoger información para:

\footnotetext{
${ }^{8}$ Esta propuesta, sostenida por varios/as investigadores/as que tratamos el tema de la identificación y las redes personales, fue asimismo presentada por Ainhoa de Federico en el artículo introductorio de un número especial sobre redes personales e identificaciones para Internacional Sociology en 2007.

${ }^{9}$ Para más información sobre esta primera etapa ver el artículo publicado en el número 6 de REDES por Aguilar, C y Molina J.L.
} 
- Analizar cómo son las relaciones personales de los jóvenes de Sarajevo para saber si siguen criterios de "división étnica", si las categorizaciones oficiales moldean realmente las auto-identificaciones o auto-percepciones, en qué casos y de qué manera.

- Analizar los procesos de identificación personal entre los jóvenes de Sarajevo, intentar ir más allá de la "identidad étnica” y recoger qué conceptos utilizan para identificarse e identificar a las personas de su red personal.

- Analizar las características de las personas que no siguen el discurso mayoritario y se niegan a identificarse mediante las categorías étnicas excluyentes.

\subsection{Metodología}

La metodología utilizada para lograr los objetivos mencionados es por un lado trabajo etnográfico, que incluye observación participante y entrevistas, recogido en un diario de campo. Por el otro hemos elaborado un cuestionario para recoger la información concreta que necesitamos para analizar las redes personales de los informantes usando una metodología de Redes Sociales.

El cuestionario, elaborado con el programa Egonet, desarrollado por McCarty (2003), consta de dos partes.

En la primera planteamos una serie de preguntas personales (unas 50) al informante (lo llamaremos EGO pues es la terminología que se utiliza al hablar de redes personales o egocéntricas) entre las que incluimos algunas preguntas referentes a la auto-identificación de EGO como las siguientes:

- Te identificas (te sientes parte) con una de las siguientes comunidades? (respuestas; Serbios, Croatas, Musulmanes/bosníacos, yo no me identifico con estas categorías).

- Te voy a pedir que escribas TRES localidades (pueblo, ciudad, etc) regiones, países u otros territorios (como Europa) o comunidades (religiosa, profesional, amistad, etc) que están relacionadas con tu vida, a las que te sientas ligado/a o que consideres importantes para definirte o identificarte.

- Escribe lo que primero responderías en la frase "yo soy...". 
En la segunda recogemos toda la información necesaria para elaborar la red personal del informante. Técnicamente se trata de elaborar una lista de 45 personas conocidas de Ego. McCarty (2002) sugiere que, tratándose de una lista libre de personas de todas las categorías, 45 son suficientes, ya que el hecho de ser una lista de libre generación tiende a generar los lazos íntimos al principio pero es suficientemente larga para obtener información de las diferentes áreas de la estructura de la red personal (lazos débiles).

A continuación, de cada una de las 45 personas se preguntan una serie de atributos como género, edad, lugar de residencia, lugar de nacimiento, nivel de estudios, ocupación, religión, intensidad de la relación (desde íntimos hasta conocidos), tipo de relación (familia, amigos, trabajo, etc.) y tres preguntas sobre identificación:

- ¿Forma el/ella parte de una de las siguientes comunidades? Respuestas: Serbios, Croatas, Musulmanes/Bosníacos, estas categorías no tienen sentido para mi.

En relación contigo, sientes que él/ella:

Forma parte de otra comunidad y diferente de mi.

Forma parte de otra comunidad pero no considero que es diferente.

No me doy cuenta de que es de otra comunidad.

Forma parte de mi comunidad.

Forma parte de mi comunidad pero es diferente de mí.

- Estas diferencias no tienen sentido para mi.

- ¿Cómo crees que el/ella te considera a ti? Respuestas: Serbio, Croata, Musulmán/Bosníaco, estas categorías no tienen sentido para él/ella.

Por último se pregunta quién conoce a quién de entre los 45 , información que resultará básica para poder elaborar la red personal y obtener medidas estructurales.

Una vez procesada la información recogida y obtenida la red personal del informante, el último paso es mostrar la red al informante para recoger la información que surge de la visualización de la red personal y los atributos de las personas de la red.

Esta última parte resulta clave pues durante la entrevista que sigue a la visualización se recoge una información de carácter cualitativo relevante para poder entender los argumentos que guían la elección de una u otra respuesta del cuestionario (Aguilar 2005). Diversas investigaciones analizan el uso de la representación grafica de la red personal en la investigación (McCarty et al. 2007, Maya Jariego et al. 2005), en ellas se constata que la visualización de las redes 
personales con el informante proporciona información adicional y detalles de la realidad social del informante que de otra manera difícilmente podrían obtenerse.

\subsection{Características de los informantes}

Mediante este cuestionario hemos entrevistado a 43 jóvenes adultos de entre 20 y 40 años, la mayoría de los cuales (un 84\%) se sitúa entre los 20 y los 30 años de edad. Respecto al nivel educativo, un $37 \%$ tiene nivel de educación secundaria, un $47 \%$ Universitaria y un $16 \%$ son postgraduados.

Si clasificamos a los informantes según la religión, tanto si son practicantes como si no lo son, tenemos un $7 \%$ de católicos, un $2 \%$ ortodoxos, un $67 \%$ musulmanes, un $16 \%$ se declaran ateos y $7 \%$ profesan otra religión. Estos datos sobre religión requieren una explicación un poco más extensa. Como hemos apuntado anteriormente Sarajevo ha dejado de ser la ciudad cosmopolita que era para albergar una amplia mayoría musulmana. Es lógico entonces que siguiendo una metodología de "bola de nieve" la mayoría de la gente con la que nos hemos ido encontrando sea de religión musulmana. De todas maneras, analizando los datos en profundidad hemos visto que tanto los ateos como los "otros" provienen de familias que no eran de origen musulmán. Haría falta analizar este dato, pero una posible hipótesis sería que en una sociedad de mayoría musulmana la autodenominación como "ateo" puede ser más "práctica” o útil para los no musulmanes.

Los matrimonios mixtos, como hemos comentado, eran comunes entre los ciudadanos de Sarajevo. Entre los informantes un $16 \%$ son hijos de matrimonios mixtos y un $25 \%$ son miembros de familias mixtas, es decir, hay gente en su familia de diferente religión pero no necesariamente los padres.

Encontramos a continuación un primer dato interesante (ver tabla 1). El 37\% de las personas entrevistadas no le encuentran sentido a auto-identificarse de manera "étnica". Para una sociedad "fuertemente dividida" como la Bosnia, por lo menos de acuerdo con los discursos oficiales, es un dato a tener en cuenta.

Resulta entonces importante ahora analizar las características de estas personas que se resisten a adoptar el discurso dominante y las categorías impuestas por las instituciones en el poder. 


\begin{tabular}{|c|c|c|c|c|c|}
\hline \multicolumn{2}{|c|}{} & Frecuencia & Porcentaje & $\begin{array}{c}\text { Porcentaje } \\
\text { válido }\end{array}$ & $\begin{array}{c}\text { Porcentaje } \\
\text { acumulado }\end{array}$ \\
\hline Válidos & Serbia & 1 & 2,3 & 2,3 & 2,3 \\
\cline { 2 - 6 } & Croata & 4 & 9,3 & 9,3 & 11,6 \\
\cline { 2 - 6 } & $\begin{array}{c}\text { Bosníaca/ M } \\
\text { usulmana }\end{array}$ & 22 & 51,2 & 51,2 & 62,8 \\
\cline { 2 - 6 } & $\begin{array}{c}\text { No tiene } \\
\text { sentido }\end{array}$ & 16 & 37,2 & 37,2 & 100,0 \\
\cline { 2 - 6 } & Total & 43 & 100,0 & 100,0 & \\
\hline
\end{tabular}

Tabla 1. Etnicidad de EGO.

\subsection{Análisis}

Para empezar a analizar las características de estas personas una tabla de contingencia (Tabla 2) entre la etnicidad y la religión nos muestra que las personas que no se identifican étnicamente son mayoritariamente ateos, otros y algunos musulmanes que se declaran no practicantes.

En BiH la religión y la etnicidad se hallan íntimamente ligadas, por lo que una manera de no ser clasificado en uno de los tres grupos étnicos es declararse ateo. Como hemos mencionado anteriormente, parece que en la nueva sociedad de Sarajevo los musulmanes tienen menos necesidad de "ocultar" su religión y les basta describirse como no practicantes separando así la religión de la etnicidad.

\begin{tabular}{|c|c|c|c|c|c|c|}
\hline & & \multicolumn{4}{|c|}{ Etnicidad } & \multirow[b]{2}{*}{ Total } \\
\hline & & Serbia & Croata & $\begin{array}{c}\text { Bosníaca/ } \\
\text { Musulmana }\end{array}$ & $\begin{array}{l}\text { No tiene } \\
\text { sentido }\end{array}$ & \\
\hline \multirow{5}{*}{$\begin{array}{l}\text { Religión } \\
\text { ego }\end{array}$} & Catolica & 0 & 3 & 0 & 0 & 3 \\
\hline & Ortodoxa & 1 & 0 & 0 & 0 & 1 \\
\hline & Musulmana & 0 & 0 & 21 & 8 & 29 \\
\hline & Atea & 0 & 1 & 0 & 6 & 7 \\
\hline & Otra & 0 & 0 & 1 & 2 & 3 \\
\hline \multicolumn{2}{|l|}{ Total } & 1 & 4 & 22 & 16 & 43 \\
\hline
\end{tabular}

Tabla 2. Tabla de contingencia Religión de EGO y Etnicidad de EGO.

Asimismo, como podemos ver en la siguiente tabla, encontramos una fuerte relación entre la práctica religiosa y la etnicidad. Mientras que la mayoría de las personas que no se identifican étnicamente no son practicantes, los que se identifican étnicamente y en mayor medida los que consideran su etnicidad muy importante son en su mayoría practicantes. 


\begin{tabular}{|c|c|c|c|c|c|}
\hline \multirow{2}{*}{} & \multicolumn{2}{|c|}{ Escala de importancia étnica } & \multirow{2}{*}{ Total } \\
\cline { 3 - 6 } \multicolumn{2}{|c|}{} & No id. étnica & Id. étnica & $\begin{array}{c}\text { Id. étnica } \\
\text { muy imp. }\end{array}$ & \\
\hline \multirow{2}{*}{$\begin{array}{c}\text { Práctica } \\
\text { religiosa }\end{array}$} & No práctica & 14 & 4 & 1 & $\mathbf{1 9}$ \\
\cline { 2 - 6 } & Práctica & 3 & 14 & 7 & $\mathbf{2 4}$ \\
\hline Total & & $\mathbf{1 7}$ & $\mathbf{1 8}$ & $\mathbf{8}$ & $\mathbf{4 3}$ \\
\hline
\end{tabular}

Tabla 3. Tabla de contingencia entre la práctica religiosa de EGO y la Escala de importancia de la Etnicidad.

De cara a seguir estudiando las características de los resistentes al discurso dominante, hemos elaborado una escala utilizando la pregunta sobre la etnicidad y las siguientes sobre la importancia de la etnicidad.

Así tenemos los que no se identifican étnicamente, que son un $40 \%$, los que se identifican étnicamente (un $42 \%$ ), y las personas para las que la identidad étnica es muy importante (un 19\%)

\begin{tabular}{|c|c|c|c|c|}
\hline & Frecuencia & Porcentaje & P. válido & P. acumulado \\
\hline No id. etnica & 17 & 39.5 & 39.5 & 39.5 \\
\hline Id. Etnica & 18 & 41.9 & 41.9 & 81.4 \\
\hline $\begin{array}{c}\text { Id. etnica muy } \\
\text { imp }\end{array}$ & 8 & 19.6 & 19.6 & 100 \\
\hline Total & $\mathbf{4 3}$ & $\mathbf{1 0 0}$ & $\mathbf{1 0 0}$ & \\
\hline
\end{tabular}

Tabla 4. Escala de importancia de la Etnicidad.

Con esta escala como variable dependiente y algunas de las variables dependientes hemos realizado diversos análisis para comprobar que factores influyen en el modo de identificarse.

Así hemos podido comprobar que las personas que no se identifican étnicamente son en general más jóvenes y tienen un salario más elevado, aunque mayoritariamente no se debe al hecho de trabajar en una organización internacional. Son personas que han vivido en el extranjero por algún periodo de tiempo más o menos largo, generalmente durante la guerra, hecho que puede influir en su forma de comprender y conceptuar el conflicto y asimismo la identidad.

Respecto a su red, el hecho más importante es que se trata de redes mayoritariamente heterogéneas en términos religiosos, es decir cuentan entre sus relaciones con personas de diversa procedencia religiosa y un alto número de ateos. Como consecuencia, el número de musulmanes en sus redes es menor. A la hora de clasificar a las personas de su red suelen identificar en mayor medida a sus 
alteri o conocidos sin utilizar las etiquetas étnicas, de igual manera que lo hacen consigo mismos.

\section{Cambio en las redes}

Algunas de las personas que entrevistamos en el primer trabajo de campo y cuyas redes analizamos en aquella primera ocasión participaron también en el segundo estudio. Gracias a ello podemos hacer un pequeño estudio para analizar el cambio en las redes personales. Entre una y otra toma de datos han pasado dos años.

A pesar de que el número de redes analizadas que coinciden en un estudio y en otro es reducido ( 7 informantes) nos pueden servir para analizar algunos aspectos interesantes del cambio en las redes.

En primer lugar resulta interesante que en un contexto que en principio podría parecer bastante estático, es decir, no se trata de inmigrantes en un proceso de integración (o no) en una cultura de acogida ni de personas cuya situación personal esté en una situación de cambio, las redes personales cambian de manera significativa en tan solo dos años. Dos personas presentan un cambio alto en sus redes personales con más de 30 personas nuevas en su red. Cuatro personas presentan un cambio medio, aproximadamente la mitad de las personas de su red son nuevas y tan solo una persona presenta un cambio bajo con 12 personas nuevas ${ }^{10}$.

Respecto a la composición y estructura de las redes personales hemos visto que la estructura es variable y en aproximadamente la mitad de los casos (4) encontramos un cambio en la estructura en tres casos de concentración de la red y en otro de segmentación de la red. Por el contrario en 6 de los 7 casos la composición religiosa de la red se mantiene invariable, tanto si se trataba de una red homogénea como de una red heterogénea a pesar del cambio en las personas concretas el tipo de composición de la red no varía.

Hemos cruzado los datos de composición y estructura con los de identificación étnica para generar nueva información de interés. Ni en el nivel de cambio ni en la estructura encontramos datos relevantes que comentar. En cambio respecto a la composición, la única persona que presenta cambio de composición es también el

\footnotetext{
10 Entendemos aquí, como en el estudio presentado por Javier Ávila en este mismo número, que un cambio alto es en el que de 30 a 45 personas son nuevas, un cambio medio es en el que 15 a 30 personas son nuevas y un cambio bajo en el que de 1 a 15 personas son nuevas.
} 
único cuya identificación étnica, que en su caso es croata, es muy importante para su identidad. El cambio de composición que presenta en su red va en el sentido de apoyar su identificación, es decir si en la red de 2003 tenia un 38\% de católicos en el 2005 los católicos son un $48 \%$ que en una sociedad mayoritariamente musulmana como es la de Sarajevo es un número muy significativo.

\section{Conclusiones}

A modo de conclusión vamos a apuntar tres ideas que, apoyadas en los datos presentados, refuerzan nuestra propuesta de identidad relacional presentada anteriormente.

En primer lugar, hemos podido observar que la identificación personal basada en la etnicidad es tan sólo una opción entre otras. Podemos encontrar en Sarajevo personas que se resisten a seguir el discursos hegemónico y dominante de división obligatoria en tres grupos étnicos.

A pesar de la fuerza que pueda tener un discurso dominante, determinadas características personales y sobre todo determinadas características de la red de relaciones personales pueden tener influencia en nuestra manera de identificarnos. En otras palabras, la identificación y la red de relaciones personales están fuertemente interrelacionadas. Las personas con las que alguien se relaciona van a contribuir a la identificación de la persona y asimismo la identificación personal influye en la elección de las amistades y relaciones. Por ejemplo, una persona con una identificación no etnicista estará más abierta a relacionarse con personas de otras religiones pues probablemente la religión del otro es un dato que no tiene en cuenta para elegir sus amistades. Una posible explicación de este fenómeno es la que nos propone Burt, la gente que hace de puente entre grupos diversos, que tiene por lo tanto acceso a diferentes discursos y cosmovisiones, tiene más opciones de elegir y por ello va a utilizar en menor medida el discurso hegemónico para auto identificarse (Burt, 2004).

El pequeño estudio de cambio en las redes también apoya nuestra contribución en este sentido. Aunque nuestras redes cambien y diversas personas entren y salgan de ellas la composición de las redes generalmente no varía y si lo hace es para reforzar este bucle que se retroalimenta entre nuestro modo de identificación y nuestras relaciones. 
Por último hemos podido ver que la religión y especialmente la práctica religiosa tienen una fuerte influencia en el proceso de identificación en Bosnia y Hercegovina.

\section{Bibliografía}

Aguilar, C (2005). "Visualización de redes personales en Sarajevo" REDES-Revista hispana para el análisis de redes sociales Vol.9, Dic 2005.

Aguilar, C; Molina, J.L. (2004). “Identidad étnica y redes personales entre jóvenes de Sarajevo", Volumen conjunto de REDES y Araucauria. Revista Iberoamericana de Filosofía, Política y Humanidades.

Anderson, Benedict (1983). Immagined Communities. London: Verso.

Banac, Ivo (1984). The national question in Yugoslavia. Ithaca and London: Cornell University Press.

Bowman, G. (1994)"Xenophobia, Fantasy and the Nation: The Logic of Ethnic Violence in Former Yugoslavia". En Victoria GODDARD, Josep LLOBERA y Chris Shore (eds) Antropology of europe: Identity and Boundaries in Conflict. Ed.. London: Berg.

Bringa, Tone (1993). "Nationality categories, national identification and identity formation in "Multinational" Bosnia". En Anthropology of east europe review, Vol 11. Nos 1-2 Autumn, 1993. Special issue: War among the yugoslavs.

Bringa, Tone (1995). Being Muslim the bosnian way. Princeton (NJ): Princeton University Press.

Brubaker, R \& COOPER, Frederick (2000). "Beyond "identity"”. Theory and Society, 29, 1-47.

Burt, Ronald (2004). "Structural Holes and Good Ideas". American Journal of Sociology, 110(2), 349-399.

Campbell, David (1998). National deconstruction. Violence, identity and justice in Bosnia. Minneapolis: University of Minessota press.

Despalatovic, E.(2000) "The roots of the war in Croatia". En Halpern, M y Kideckel, David. A (Eds.) Neighbors at war: Antropological Perspectives on Yugoslav Ethnicity, Culture and History. Pennsylvania State Press 
Donia, Robert J. y Fine, John V.A. (1994). Bosnia and Hercgovina, A tradition betrayed. London: C.Hurst\&Co

De Federico de la Rua, Ainhoa. "Networks and Identifications: A relational Approach to Social identities". International Sociology 2007; 22 683-699

Gagnon, V.P (1994). "Etnic Conflict as Demobilizer: The case of Serbia" Presentación en el congreso The Balkans: Nationalism and Ethnicity. Hamilton College, Clinton, NY, 26 Abril, 1994.

Hammel, E.A(1993). "The Yugoslav Labyrinth". En Anthropology of east europe review, Vol 11. Nos 1-2 Autumn,. Special issue: War among the yugoslavs.

Kaldor, Mary (2001). Las nuevas guerras: violencia organizada en la era global. Barcelona: Tusquets.

Lovrenovic, Ivan (2001). Bosnia: A Cultural History. London: Saqui books.

Maya J ariego, I sidro y Holgado Daniel (2005). “Lazos fuertes y proveedores múltiples de apoyo: comparación de dos formas de representación gráfica de las redes personales" Empiria №10 Julio-Diciembre 2005 p.107-127

McCarty, C; Molina, JL; Aguilar, C; Rota, L (2007)."A comparison of social network mapping and personal network visualization", Field Methods Vol. 19 (2) May (145162)

McCarty, Chris (2003). Egonet. Personal Network Software <http://survey.bebr.ufl.edu/EgoNet/> [Visited: 2-3-04].

Petrovic, Edit (2000). "Ethnonationlism and the dissolution of Yugoslavia". en Halpern, M y Kideckel, David. A (Eds.) Neighbors at War: Antropological Perspectives on Yugoslav Ethnicity, Culture and History. Pennsylvania State Press

Prosic-Dvornic, Mirjiana (2000). "Serbia: The inside story". En Halpern, M y Kideckel, David. A (Eds.) Neighbors at war: Antropological Perspectives on Yugoslav Ethnicity, Culture and History. Pennsylvania State Press

Simic, Andrei (2000). "Nationalism as a Folk Ideology: The case of former Yugoslavia". en Halpern, M y Kideckel, David. A (Eds.) Neighbors at war: Antropological Perspectives on Yugoslav Ethnicity, Culture and History. Pennsylvania State Press 
UNDP (2000). Human Development Report Bosnia and herzegovina 2000 Youth. Sarajevo: United Nations Development Programme.

Vucetic, Srdjan. (2004). "Identity is a joking matter, Intergroup Humor in Bosnia". Spacesofidentity Vol. 4.1 www.spacesofidentity. net. 